\title{
DYSLIPIDEMIA IN PATIENTS WITH ACTIVE RHEUMATIC DISEASE
}

\author{
Dr. Binit Vaidya ${ }^{1}$, Dr. Manisha Bhochhibhoya ${ }^{1}$
}

\section{ABSTRACT}

\section{INTRODUCTION:}

Rheumatic diseases have been found to be associated with an increased risk of cardiovascular disease (CVD) and dyslipidemia. Constant state of inflammation among patients with active rheumatic diseases predispose to endothelial damage leading to increased risk of dyslipidemia and CVD. Dyslipidemias are being increasingly recognized as an important contributory factor towards the development of CVD and also the leading cause of death in various autoimmune diseases. We aim to study the prevalence and pattern of dyslipidemia in these patients.

\section{MATERIALS \& METHODS:}

Consecutive patients with active rheumatic disease of $>3$ months duration and fibromyalgia attending rheumatology OPD were included from January 2013 to January 2014. Patients with diabetes, hypertension, hypothyroidism, CAD, CKD and those on statin or fibrates were excluded. Activity was determined by clinical features, ESR and CRP levels. Data were collected on predesigned excel sheet including fasting lipid profile.

\section{RESULTS:}

A total of 560 patients were enrolled (RA-255, SLE-70, other-45, FMS-190). Any form of dyslipidemia was found in $75.9 \%$ of patients. Isolated raised triglyceride $>300 \mathrm{mg} / \mathrm{dl}$ was seen in $178(41.9 \%)$ of them, whereas LDL $>120 \mathrm{mg} / \mathrm{dl}$ was seen $235(55.3 \%)$ of patients $(23.7 \%$ had LDL $>200)$. Almost all patients $(98.7 \%)$ had LDL:HDL ratio of $>2.5$. Dyslipidemia was seen in $86(45.2 \%)$ patients with fibromyalgia but none of them had LDL $>120 \mathrm{mg} / \mathrm{dl}$.

\section{CONCLUSION:}

Patients with active rheumatic disease are likely to have significant dyslipidemia which can contribute to excess cardiovascular mortality in these patients. Early identification and correction of significant lipid abnormalities may help to reduce the morbidity and mortality in these patients.

KEY WORDS: Dyslipidemia; Rheumatic diseases; Cardiovascular disease; Lipid profile.

1. National centre for Rheumatic Diseases

\section{For Correspondence}

Dr Binit Vaidya, MD, FACR

Email: drbinitvaidya@yahoo.com

Affiliation: National Centre for Rheumatic Diseases, Kathmandu 


\section{INTRODUCTION:}

Rheumatic diseases have been found to be associated with an increased risk of cardiovascular disease (CVD) and dyslipidemia. ${ }^{1}$ Various studies have been done on patients with rheumatic diseases to study the patterns of dyslipidemias. It has been observed that increased inflammation and active disease has an impact on lipid patterns in blood. The constant state of inflammation among patients with active rheumatic diseases predispose to endothelial damage leading to increased risk of dyslipidemia and CVD. ${ }^{2}$

In general population, dyslipidemia has been shown to be one of the strongest predictors of CVD, with elevated levels of low density lipoproteins (LDL) forming the primary treatment target according to national guidelines. ${ }^{3,4}$ About $54 \%$ of rheumatoid arthritis (RA)patient were found to have various types of dyslipidemias. ${ }^{1}$ They are being increasingly recognized as an important contributory factor towards the development of CVD among active rheumatic diseases and also the leading cause of death in various autoimmune diseases, mainly rheumatoid arthritis (RA), systemic lupus erythematosus (SLE), ankylosing spondylitis and antiphospholipid antibody syndrome. ${ }^{5,6}$ RA is associated with $50 \%$ increase in incidence of myocardial infarction (MI) and cardiovascular diseases as compared to general population. ${ }^{7}$ CVD is the most prominent cause of mortality and morbidity among SLE patients, prevalence ranges between $6-10 \%{ }^{8}$

In context to Nepal, dyslipidemia is quite prevalent among general population and the lipid pattern is significantly different from that seen in other Asian studies. ${ }^{9-12}$ We do not have data available on the lipid patterns of patients with active rheumatic diseases. Thus, the rationale of this study is to see the prevalence and pattern of dyslipidemias in patients with active rheumatic disorders in Nepal.

\section{METHODOLOGY:}

The study was cross sectional observational study conducted at Rheumatology OPD of National Center for Rheumatic Diseases (NCRD) and a tertiary hospital in Kathmandu from January 2013 to January 2014. Consecutive patients of all age group with active inflammatory rheumatic diseases of $>3$ months duration and fibromyalgia were included. Patients with diabetes, hypertension, hypothyroidism, CVD, chronic kidney disease and those on lipid lowering drugs (statins and fibrates) were excluded from the study. Informed consent was obtained from all subjects before encrollment in the study.

Detailed history, clinical examination and review of past medical records were performed. A predesigned excel sheet was filled to evaluate the clinical parameters used in assessment of activity of each rheumatic disease.

Laboratory investigations were sent for C-reactive protein (CRP), erythrocyte sedimentation rate (ESR), lipid profile including high density lipoproteins (HDL), low density lipoproteins (LDL), total cholesterol (TC) and triglycerides (TG) after an overnight fasting of 12 hours.

Statistical analysis was performed using SPSS 19. Simple descriptive statistics were used to define the prevalence and type of dyslipidemia in these patients.

\section{RESULTS:}

A total of 560 patients were enrolled in the study (RA 255, SLE 70, FMS 190 and others 45)[figure1].Others included ankylosing spondylitis, polymyositis, anti-phospholipid antibody syndrome, scleroderma etc.

Any form of dyslipidemia was found in $75.9 \%$ of patients. Isolated raised triglyceride (TG) $>300 \mathrm{mg} / \mathrm{dl}$ was seen in 178 $(41.9 \%)$ of them, whereas Low Density Lipoprotein (LDL) $>120 \mathrm{mg} / \mathrm{dl}$ was seen $235(55.3 \%)$ of patients $(23.7 \%$ had LDL>200). Almost all patients (98.7\%) had LDL:HDL ratio of $>2.5$. Dyslipidemia was seen in $86(45.2 \%)$ patients with fibromyalgia but none of them had LDL $>120 \mathrm{mg} / \mathrm{dl}$.

\section{DISCUSSION:}

The complexity and diversity of many rheumatological conditions is often attributed to their underlying autoimmune nature. Autoimmunity forms the basis of many rheumatological diseases, and may contribute not only to the classical clinical manifestations but also to the complications. Dyslipidemia can be considered as a major risk factor for CVD and is very common in patients with active rheumatic diseases. ${ }^{5,6}$ Many of the rheumatological diseases, including RA and SLE are associated with an excess cardiovascular morbidity and mortality. ${ }^{13-15} \mathrm{RA}$ is associated with $50 \%$ increase in incidence of myocardial infarction and cardiovascular diseases as compared to general population. ${ }^{7}$ The prevalence of CVD among SLE patients ranges between 6 $-10 \%{ }^{8}$

Active rheumatic diseases are associated with a heightened inflammatory state in varying degree. ${ }^{16}$ Due to the increased permeability of the dysfunctional endothelium, excess LDL infiltrates the artery wall and is retained in the intima. LDL then undergoes modification and oxidation, initiating an inflammatory response in the artery wall. ${ }^{17,18}$ 
Patterns of dyslipidemias in autoimmune rheumatic diseases have been studied in different parts of the world; but there is no study in Nepal on this subject. Thus, the study was conducted in Nepalese patients with various types of rheumatic diseases. Different types of lipid disorders were observed in $75.9 \%$ of the total patients. Population prevalence of dyslipidemia is around 31\% worldwide. ${ }^{4}$ Similar data was seen among Nepalese general population too. ${ }^{19,20}$ Needless to say, dyslipidemia is more common among diabetics. ${ }^{21}$ A study in 2011 has shown elevated TG levels to be associated with increased CVD risk among RA patients as compared to those without RA. ${ }^{22}$ The pattern of dyslipidemia may vary from one country to another. ${ }^{23}$ Around $42 \%$ of our patients also had isolated raised TG levels.

When considering different lipid fractions individually, low HDL and deranged cholesterol are the most significant dyslipidemias associated with disease activity among these rheumatic patients. ${ }^{1}$ Almost all (98.7\%) patients having high LDL:HDL ratio indicates towards this risk factor also.

Thus, we can summarize that dyslipidemia is more prevalent in patients with active rheumatic disease as compared to the general population. An isolated high TG level, high LDL:HDL ratio followed by high LDL levels are the main patterns seen in these patients which is comparable to diabetic patients. ${ }^{24}$

\section{CONCLUSION:}

Patients with active rheumatic disease are likely to have significant dyslipidemia which can contribute to excess cardiovascular mortality in these patients. Although the pattern and prevalence of dyslipidemia appears to differ between the autoimmune rheumatic diseases, the mechanism underlying these changes include the interplay of inflammation and autoantibody formation. Thus, early identification and correction of significant lipid abnormalities may help to reduce the morbidity and mortality in these patients.

Figure 1

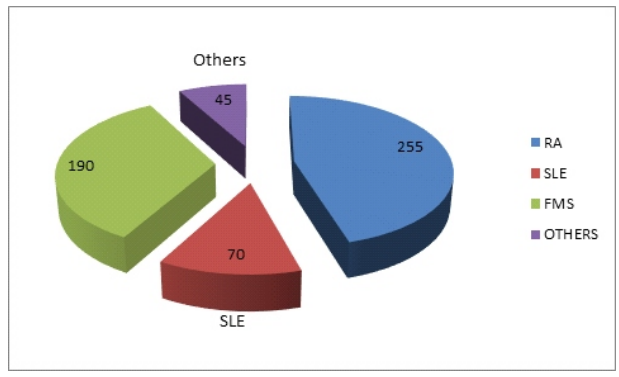

\section{REFERENCES:}

1. Nisar A, Rasheed U, Aziz W, Farooqi AZ. Prevalence of dyslipidemias in autoimmune rheumatic diseases. $J$ College Physicians Surg Pak. 2012;22(4):235-9.

2. Toms TE, Panoulas VF, Kitas GD. Dyslipidaemia in rheumatological autoimmune diseases. Open Cardiovasc Med J. 2011;5:64-75.

3. Executive Summary of The Third Report of The National Cholesterol Education Program (NCEP) Expert Panel on Detection, Evaluation, And Treatment of High Blood Cholesterol In Adults (Adult Treatment Panel III). JAMA. 2001;285(19):2486-97.

4. Iqbal SP, Dodani S, Qureshi R. Risk factors and behaviours for coronary artery disease (CAD) among ambulatory Pakistanis. $J$ PakMed Assoc. 2004;54(5):261-6.

5. Shoenfeld Y, Gerli R, Doria A, Matsuura E, Cerinic MM, Ronda $N$, et al. Accelerated atherosclerosis in autoimmune rheumatic diseases. Circulation. 2005;112(21):3337-47.

6. Bergstrom U, Jacobsson LT, Turesson C. Cardiovascular morbidity and mortality remain similar in two cohorts of patients with long-standing rheumatoid arthritis seen in 1978 and 1995 in Malmo, Sweden. Rheumatology (Oxford). 2009;48(12):1600-5.

7. Frostegard J. Atherosclerosis in patients with autoimmune disorders. Arterioscler Thromb Vasc Biol. 2005;25(9):1776-85.

8. Spellman CW. Strategies for optimizing lipid treatment outcomes. J Am Osteopath Assoc. 2003;103(1 Suppl 1):S12-5.

9. Haddad FH, Omari AA, Shamailah QM, Malkawi OM, Shehab AI, Mudabber HK, et al. Lipid profile in patients with coronary artery disease. Saudi Med J. 2002;23(9):1054-8.

10. Li Z, Yang $R, X u G$, Xia T. Serum lipid concentrations and prevalence of dyslipidemia in a large professional population in Beijing. Clin Chem. 2005;51(1):144-50.

11. Szapary PO, Rader DJ. The triglyceride-high-density lipoprotein axis: an important target of therapy? Am Heart $J$. 2004; $148(2): 211-21$.

12. Azizi F, Raiszadeh F, Salehi $P$, Rahmani M, Emami $H$, Ghanbarian A, et al. Determinants of serum HDL-C level in a Tehran urban population: the Tehran Lipid and Glucose Study. Nutr Metab Cardiovasc Dis. 2002;12(2):80-9.

13. Kitas GD, Erb N. Tackling ischaemic heart disease in rheumatoid arthritis. Rheumatology (Oxford). 2003;42(5): 607-13. 
14. Manzi S, Meilahn EN, Rairie JE, Conte CG, Medsger TA, Jr., Jansen-McWilliams L, et al. Age-specific incidence rates of myocardial infarction and angina in women with systemic lupus erythematosus: comparison with the Framingham Study. Am J Epidemiol. 1997;145(5):408-15.

15. Van Doornum S, McColl G, Wicks IP. Accelerated atherosclerosis: an extraarticular feature of rheumatoid arthritis? Arthritis Rheum. 2002;46(4):862-73.

16. Libby $P$, Ridker PM, Maseri A. Inflammation and atherosclerosis. Circulation. 2002;105(9):1135-43.

17. Skalen K, Gustafsson M, Rydberg EK, Hulten LM, Wiklund $O$, Innerarity TL, et al. Subendothelial retention of atherogenic lipoproteins in early atherosclerosis. Nature. 2002;417 (6890) :750-4.

18. Leitinger N. Oxidized phospholipids as modulators of inflammation in atherosclerosis. Curr Opin Lipidol. 2003;14(5):421-30.

19. Limbu YR, Rai SK, Ono K, Kurokawa M, Yanagida JI, Rai G, et al. Lipid profile of adult Nepalese population. Nepal Med Coll J. 2008;10(1):4-7.
20. Karki DB, Neopane A, Pradhan B, Magar A. Lipid levels in Nepalese population. Kathmandu Univ Med $J$ (KUMJ). 2004;2(4):349-53.

21. Dixit AK, Dey R, Suresh A, Chaudhuri S, Panda AK, Mitra A, et al. The prevalence of dyslipidemia in patients with diabetes mellitus of ayurveda Hospital. J Diabetes Metab Disord. 2014;13:58.

22. Myasoedova E, Crowson CS, Kremers HM, Roger VL, FitzGibbon PD, Therneau TM, et al. Lipid paradox in rheumatoid arthritis: the impact of serum lipid measures and systemic inflammation on the risk of cardiovascular disease. Ann Rheum Dis. 2011;70(3):482-7.

23. Cesur M, Ozbalkan Z, Temel MA, Karaarslan Y. Ethnicity may be a reason for lipid changes and high Lp(a) levels in rheumatoid arthritis. Clin Rheumatol. 2007;26(3):355-61.

24. Mooradian AD. Dyslipidemia in type 2 diabetes mellitus. Nat Clin Pract Endocrinol Metab. 2009;5(3):150-9. 